Review began 01/23/2022 Review ended 01/26/2022 Published 02/02/2022

(c) Copyright 2022

Beheshti et al. This is an open access article distributed under the terms of the Creative Commons Attribution License CCBY 4.0., which permits unrestricted use, distribution, and reproduction in any medium, provided the original author and source are credited.

\section{Atypical Stevens-Johnson Syndrome Associated With Mycoplasma Pneumoniae}

\author{
Ramin Beheshti ${ }^{1}$, Bryan Cusack ${ }^{1}$ \\ 1. Pediatrics, Penn State Health Milton S. Hershey Medical Center, Hershey, USA
}

Corresponding author: Ramin Beheshti, rbeheshti@pennstatehealth.psu.edu

\begin{abstract}
Mycoplasma pneumoniae primarily causes atypical pneumonia in children and young adults. $7 \%-8 \%$ of patients with $M$. pneumoniae infections may experience extra-pulmonary manifestations, including $M$. pneumoniae-associated Stevens-Johnson Syndrome (SJS), also known as atypical SJS. In recent literature, there have been a few reports of isolated mucositis in children with $M$. pneumoniae infections. Due to significant overlap with several diseases, including autoimmune disease and infections, atypical mucositis associated with $M$. pneumoniae is often a diagnostic challenge. In addition, due to limited cases of $M$. pneumoniae-associated SJS, there is no established standardized treatment guideline that has been shown to reduce hospitalization duration and/or disease progression associated with $M$. pneumoniae-associated SIS. We report a case of isolated mucositis in the absence of cutaneous involvement in a 10-year-old patient with an acute $M$. pneumoniae infection. Examination revealed erythematous ulcerations of his lips and pharynx with patchy exudates and bilateral submandibular lymphadenopathy. Laboratory investigation revealed a negative respiratory polymerase chain reaction (PCR) panel, which included M. pneumoniae. Further testing revealed a positive $M$. pneumoniae immunoglobulin M (IgM) titer on enzyme immunoassay. The diagnosis of atypical SJS was made secondary to M. pneumoniae. Treatment was initiated with systemic steroids and oral antibiotics. Limitations in diagnostic testing for M. pneumoniae in combination with non-specific clinical presentation make for challenges in confirming this pattern of SJS due to a primary M. pneumoniae infection. In this case, serological testing confirmed our suspected diagnosis, which guided treatment and helped reveal some of the difficulties in diagnosing and managing $M$. pneumoniae-associated SJS.
\end{abstract}

Categories: Dermatology, Pediatrics, Infectious Disease

Keywords: delayed diagnosis, diagnostic medicine, atypical rash, mycoplasma pneumonia, steven johnson syndrome

\section{Introduction}

Mycoplasma pneumoniae is a common infectious cause of community-acquired pneumonia; however, isolated mucositis has been reported in children with M. pneumoniae infections [1]. SJS is a type four hypersensitivity reaction to cutaneous and mucosal tissue involving the stimulation of cytotoxic CD8+ T cells and helper CD4+ T cells [2,3]. Drug reactions account for 50\% of typical cases, which usually present with targeted skin rash in addition to mucositis [3]. Recently several reports of isolated mucositis (atypical SJS) have been reported with infections [4]. One such causative agent of atypical SJS is $M$. pneumoniae [5]. M. pneumoniae is an obligate intercellular organism associated with pneumonia in pediatric and young adult populations [1]. In addition to respiratory involvement $7 \%-8 \%$ of cases result in extrapulmonary manifestation, including arthritis (<3\%), hepatitis $(<5 \%)$, pericarditis $(<1 \%)$, hemolytic anemia (<5\%), aseptic meningitis $(<1 \%)$, and SJS $(<1 \%)[6]$. M. pneumoniae-associated SJS (atypical SJS) is clinically diagnosed based on the distribution of the rash, along with associated clinical findings [4]. Typical SJS typically begins with a fever and progresses with non-specific symptoms that may overlap with various infectious or inflammatory processes [4]. As it progresses, patients may experience mucosal desquamation around the mouth and anogenital regions [7]. In typical SJS, cutaneous involvement usually includes the face, arms, legs, and back and appears in the form of targetoid lesions [4]. Typical SJS is a clinical diagnosis; however, in atypical SJS sources of infectious etiology may be elicited, with further laboratory workup to mitigate timely treatment. Serology is the gold standard test for laboratory diagnosis [8]. Polymerase chain reaction (PCR) is a rapid and helpful test as well, especially when combined with serology [8]. Clinicians often rely on PCR testing to exclude $M$. pneumoniae due to the rapid availability of results; however, this test has a sensitivity of $60 \%-70 \%$, which may result in false negatives [8]. Serological testing has a sensitivity of $90 \%$. Unfortunately, serological testing is often excluded in such presentations as the non-specific clinical presentations may often mislead clinicians to work up a wide spectrum of diseases that affect the mucous membranes. In addition, M. pneumoniae-associated mucositis is extremely rare, so limitations in awareness of $M$. pneumoniae-associated mucositis may lead to delayed testing [8]. Therefore, appropriate management and treatment may be delayed due to diagnostic challenges. Therefore, early diagnosis is important in guiding the management and treatment of the underlying infectious cause for SJS. In addition to diagnostic challenges, there is currently no established standardized guideline to help clinicians manage $M$. pneumoniae-associated SJS [8]. Antibiotics may help treat M. pneumoniae infections, but no clear relationship between antibiotics and progression of mucositis has been established [9]. The use of steroids in M. pneumoniae-associated SIS is controversial, with no evidence of reduced mortality or hospital duration based on current literature [8]. Supportive care, including nutrition optimization and pain management, has 


\section{Cureus}

been associated with improved patient satisfaction but has not been associated with reduced hospitalization duration [9]. We report a case of isolated mucositis in the absence of cutaneous involvement in a pediatric patient with $M$. pneumoniae. We demonstrate difficulties in making this diagnosis due to a non-specific clinical presentation and the insensitivity of the most utilized methods of detecting M. pneumoniae. We also highlight the absence of a standardized guideline for the management of patients with M. pneumoniaeassociated SIS and discuss the outcome of our patient's specific treatment regimen.

\section{Case Presentation}

A 10-year-old male with no significant past medical history initially presented with 1-2 days of bilateral conjunctivitis and mucositis with intermittent fevers for two weeks. Fevers had not occurred daily but had not resided despite daily antipyretics. Following the initial onset of fevers, he developed a dry cough, congestion, and rhinorrhea. He had not experienced increased work of breathing or wheezing. His parents reported no history of asthma. He developed mouth sores with severe odynophagia 1-2 days before admission. He had no skin rash or extremity/facial swelling. He was not taking any scheduled prescription medications at home. On physical examination, his vitals are as follows: temperature, 97.8F (36.5C); blood pressure, 84/55 $\mathrm{mm} \mathrm{Hg}$; heart rate, 122 beats/min; respiratory rate, 27 breaths/min. Examination revealed erythematous ulcerations of his lips and pharynx with patchy exudates and bilateral submandibular lymphadenopathy (Figure 1). In addition, he demonstrated bilaterally injected conjunctiva with purulent drainage. No skin lesions or rashes were present. The rest of his examination findings are unremarkable. Laboratory investigation revealed a negative respiratory PCR panel that tested for common viruses and bacteria, including $M$. pneumoniae. Additionally, laboratory investigation revealed elevated C-Reactive protein at $3.1 \mathrm{mg} / \mathrm{dL}$ (normal range: $<10 \mathrm{mg} / \mathrm{dL}$ ) and erythrocyte sedimentation rate at $58 \mathrm{~mm} / \mathrm{hr}$ (normal range: $3-13 \mathrm{~mm} / \mathrm{hr}$ ) and an unremarkable complete blood count (CBC). Further testing revealed a positive $M$. pneumoniae IgM titer on enzyme immunoassay, which resulted on day three of his hospitalization. The diagnosis of atypical Stevens-Johnson Syndrome (SJS) was made secondary to M. pneumoniae. The patient was started on a seven-day course of IV Methylprednisolone at $1 \mathrm{mg} / \mathrm{kg} /$ day divided by four daily doses and a five-day course of Azithromycin at $500 \mathrm{mg}$ orally on day one, followed by $250 \mathrm{mg}$ on days 2-5. His symptoms improved quickly and resolved three days after the onset of treatment. He was discharged home upon completion of IV steroids with complete resolution of his mucositis.

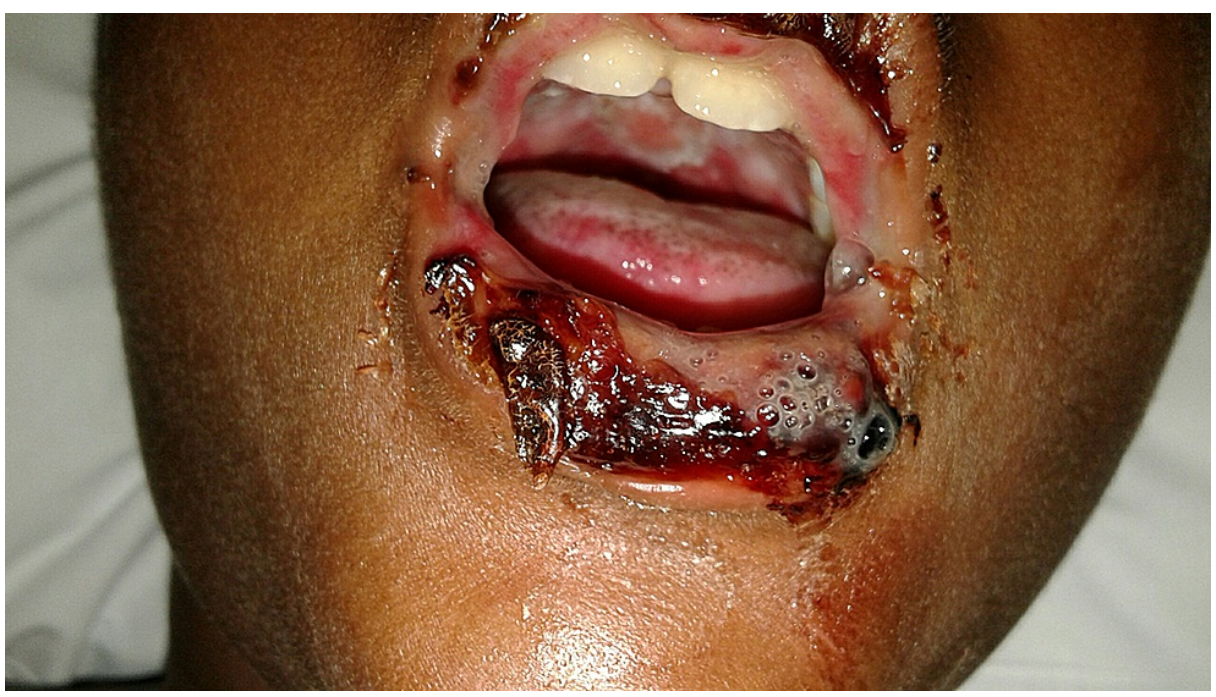

\section{FIGURE 1: Initial examination revealing erythematous ulcerations of the lips and pharynx with patchy exudates}

\section{Discussion}

SIS is often characterized by skin, mucosal, and genitourinary lesions [2]. This case presents a unique atypical presentation of SJS characterized by isolated oral mucositis in the absence of cutaneous lesions. This atypical presentation of SJS has been linked to M. pneumoniae, which has been reported as an extrapulmonary manifestation in children and young adults aged 6-19 [1]. Extrapulmonary manifestations of $M$. pneumoniae are rare but have been reported in previous literature [1]. M. pneumoniae-associated SJS without cutaneous involvement is extremely rare and poorly understood. Establishing the diagnosis of atypical SJS is challenging due to clinical similarities with more common diseases, including autoimmune diseases and infections. Additional infectious agents, including HSV, group A streptococcus, EBV, and Hepatitis B virus, have been associated with isolated mucositis [1]. Limitations in diagnostic testing for M. pneumoniae in combination with non-specific clinical presentation make for challenges in confirming this pattern of SJS due to a primary M. pneumoniae infection [8]. Given the association between SIS and M. pneumoniae, serological diagnostic testing for M. pneumoniae should be considered in pediatric patients with isolated 
mucositis, particularly in cases where the clinical diagnosis of SJS may be more uncertain. Two factors should be evaluated when choosing the best diagnostic test, accuracy and time to obtain results. $M$. pneumoniae is a small ubiquitous microorganism and lacks a cell wall, making it difficult to detect by gram staining [1]. Cultures can confirm their presence but take 2-6 weeks, making them ineffective in guiding treatment during an acute presentation [10]. PCR is a quick diagnostic method but has a sensitivity of $60 \%-$ $70 \%$, leading to a high rate of false negatives as with our patient. Serology testing specifically complements fixation assays, and indirect immunofluorescence has better sensitivity and specificity, 80\%-90\% and 92\%$100 \%$, respectively [8]. However, this method takes several days and can increase the cost of hospitalization [8]. There is currently no established standardized guideline to help clinicians manage M. pneumoniaeassociated SJS [8]. Often antibiotics are initiated to treat $M$. pneumoniae, but the dosing and duration are not well established. The use of steroids in M. pneumoniae-associated SJS is controversial, with no known studies that have demonstrated reduced mortality or hospital duration with the initiation of steroids. Supportive care, including nutrition optimization and pain management, has been associated with improved patient satisfaction but has not been associated with reduced hospitalization duration [8].

Therefore, treatment of M. pneumoniae-associated SJS remains poorly understood, with no objective data to provide evidence-based guidelines for clinicians to manage such patients. In this case, serological testing confirmed our suspected diagnosis and guided effective treatment. We highlight that the symptoms resolved three days after the initiation of a seven-day course of systemic steroids (Methylprednisolone) and a fiveday course of oral antibiotics (azithromycin). We suggest that when initiated early in disease progression, the combination of steroids and antibiotics may reduce the duration of hospitalization and improve clinical outcomes for patients with $M$. pneumoniae-associated mucositis, as in our case.

\section{Conclusions}

Atypical SJS differs from classical SIS with regards to clinical presentation and etiology. M. pneumoniaeassociated SJS has been reported in the literature but is extremely rare and therefore poorly understood. $M$. pneumoniae-associated SJS can mimic various autoimmune and infectious conditions, which makes this a diagnostic challenge. Currently, no established treatment regimen has been shown to reduce hospitalization duration and/or mortality associated with $M$. pneumoniae-associated SJS. This case outlines the diagnostic challenges in identifying $M$. pneumoniae as the causative agent of atypical SJS and demonstrates shortened hospital duration and reduced disease progression following the initiation of methylprednisolone and azithromycin. Cases that demonstrate reduced hospitalization duration and disease progression with timely diagnosis and effective treatment may improve objective data and help establish a standardized, evidence-based treatment guideline with guidance on how to care for patients with M. pneumoniaeassociated SJS.

\section{Additional Information \\ Disclosures}

Human subjects: All authors have confirmed that this study did not involve human participants or tissue. Conflicts of interest: In compliance with the ICMJE uniform disclosure form, all authors declare the following: Payment/services info: All authors have declared that no financial support was received from any organization for the submitted work. Financial relationships: All authors have declared that they have no financial relationships at present or within the previous three years with any organizations that might have an interest in the submitted work. Other relationships: All authors have declared that there are no other relationships or activities that could appear to have influenced the submitted work.

\section{References}

1. Schalock PC, Dinulos JG: Mycoplasma pneumoniae-induced cutaneous disease. Int J Dermatol. 2009, 48:67380; quiz 680-1. 10.1111/j.1365-4632.2009.04154.x

2. Ravin KA, Rappaport LD, Zuckerbraun NS, Wadowsky RM, Wald ER, Michaels MM: Mycoplasma pneumoniae and atypical Stevens-Johnson syndrome: a case series. Pediatrics. 2007, 119:e1002-5. 10.1542/peds.20062401

3. Léauté-Labrèze C, Lamireau T, Chawki D, Maleville J, Taïeb A: Diagnosis, classification, and management of erythema multiforme and Stevens-Johnson syndrome. Arch Dis Child. 2000, 83:347-52. 10.1136/adc.83.4.347

4. Frey N, Bodmer M, Bircher A, Jick SS, Meier CR, Spoendlin J: Stevens-Johnson syndrome and toxic epidermal necrolysis in association with commonly prescribed drugs in outpatient care other than antiepileptic drugs and antibiotics: a population-based case-control study. Drug Saf. 2019, 42:55-66. 10.1007/s40264-018-0711-X

5. Latsch K, Girschick HJ, Abele-Horn M: Stevens-Johnson syndrome without skin lesions. J Med Microbiol. 2007, 56:1696-9. 10.1099/jmm.0.47318-0

6. Vercueil A, Walsh S: Extracutaneous manifestations and long-term sequelae of Stevens-Johnson syndrome/toxic epidermal necrolysis. Br J Dermatol. 2015, 172:312. 10.1111/bjd.13631

7. Catt CJ, Hamilton GM, Fish J, Mireskandari K, Ali A: Ocular manifestations of Stevens-Johnson syndrome and toxic epidermal necrolysis in children. Am J Ophthalmol. 2016, 166:68-75. 10.1016/j.ajo.2016.03.020

8. Hammerschlag MR: Mycoplasma pneumoniae infections. Curr Opin Infect Dis. 2001, 14:181-6. 10.1097/00001432-200104000-00012

9. Brüggen MC, Le ST, Walsh S, et al.: Supportive care in the acute phase of Stevens-Johnson syndrome and toxic epidermal necrolysis: an international, multidisciplinary delphi-based consensus. Br J Dermatol. 2021, 


\section{Cureus}

185:616-26. 10.1111/bjd.19893

10. Young L, Sung J, Stacey G, Masters JR: Detection of Mycoplasma in cell cultures . Nat Protoc. 2010, 5:929-34. 10.1038/nprot.2010.43 